\title{
Closed-Form Sum-MSE Minimization for the Two-User Gaussian MIMO Broadcast Channel
}

\author{
Johannes Kron, Daniel Persson, Mikael Skoglund and Erik G. Larsson
}

\section{Linköping University Post Print}

N.B.: When citing this work, cite the original article.

(C2011 IEEE. Personal use of this material is permitted. However, permission to reprint/republish this material for advertising or promotional purposes or for creating new collective works for resale or redistribution to servers or lists, or to reuse any copyrighted component of this work in other works must be obtained from the IEEE.

Johannes Kron, Daniel Persson, Mikael Skoglund and Erik G. Larsson, Closed-Form SumMSE Minimization for the Two-User Gaussian MIMO Broadcast Channel, accepted IEEE Communications Letters.

Postprint available at: Linköping University Electronic Press

http://urn.kb.se/resolve?urn=urn:nbn:se:liu:diva-70179 


\title{
Closed-Form Sum-MSE Minimization for the Two-User Gaussian MIMO Broadcast Channel
}

\author{
Johannes Kron, Daniel Persson, Mikael Skoglund, and Erik G. Larsson
}

\begin{abstract}
We study the Gaussian multiple-input, multipleoutput broadcast channel, where a base station with $N_{T}$ antennas transmits $K$ independent messages to $K$ users, each having a single receive antenna. The messages consist of independent, identically distributed Gaussian random variables and we study linear transmission with an end-to-end distortion criterion. By using an already established uplink/downlink duality and a recently discovered special relation between beamforming vectors and channel vectors, we present a closed-form expression for the optimal power allocation in the two-user case. We also outline an iterative algorithm that finds the optimal power allocation for an arbitrary number of users.
\end{abstract}

Index Terms-MIMO systems, mean square error, linear transmission, power allocation.

\section{INTRODUCTION}

$\mathbf{W}$ E consider the Gaussian multiple-input, multipleoutput (MIMO) broadcast channel, where a base station with $N_{T}$ antennas transmits $K$ independent messages to $K$ users, each having a single receive antenna. The messages consist of independent, identically distributed (i.i.d.) complex Gaussian random variables and we study transmission with an end-to-end distortion criterion. The capacity region of the Gaussian MIMO broadcast channel is achievable with dirty-paper precoding (DPC) [1]. Since the messages are independent, we could combine an optimal source code with DPC, achieving the distortion-rate bound for each message, to yield an optimal scheme. However, for the source code and DPC to be optimal, it is in general required that infinite block lengths are used. Motivated by low-delay and low-complexity constraints, we instead turn to analog transmission using linear precoding, where the problem is to determine the optimal beamforming vector and power allocation to use for each user's message.

Linear precoding is a well-studied topic. The difficulty in solving the problem lies in the fact that the optimal beamforming vector to each user is dependent both on the power allocation and also on the beamforming vectors used to all other users. Especially interesting to us are the results on signal-to-interference-and-noise ratio (SINR) balancing [2],

Manuscript received March 15, 2011. The associate editor coordinating the review of this letter and approving it for publication was H. Liu.

This work has been supported in part by the Swedish Governmental Agency for Innovation Systems (VINNOVA), the Swedish Research Council (VR), and the Swedish Foundation for Strategic Research (SSF). E. Larsson is a Royal Swedish Academy of Sciences (KVA) Research Fellow supported by a grant from the Knut and Alice Wallenberg Foundation.

J. Kron and M. Skoglund are with the School of Electrical Engineering and the ACCESS Linnaeus Center, Royal Institute of Technology (KTH), SE-100 44 Stockholm, Sweden (e-mail: \{johk, skoglund\}@ee.kth.se).

D. Persson and E. G. Larsson are with the Department of Electrical Engineering, Linköping University, SE-581 83 Linköping, Sweden (e-mail: \{danielp, egl\}@isy.liu.se).

Digital Object Identifier 10.1109/LCOMM.2011.11.110571 where an uplink/downlink duality is shown to exist. By using this duality, it is shown that one can determine the optimal beamforming vectors by first solving the much simpler dual uplink problem, which includes uplink power allocation and receive beamforming vectors. The uplink problem is simpler because the beamforming vector for each user is independent of the beamforming vectors for the other users. The optimal downlink solution is obtained by using the dual uplink receive beamforming vectors as transmit beamforming vectors and next finding the optimal downlink power allocation. The distortion criterion we will use is the mean-squared error (MSE), which has a close relation to the SINR. The uplink/downlink duality has been shown to also apply to the MSE region [3].

Algorithms based on convex optimization and iterative techniques for determining optimal power allocation and beamforming vectors were proposed in [2] and [3], [4] for the case of SINR and MSE, respectively. The main contribution of this letter is a closed-form solution for the optimal uplink/downlink MSE power allocation in the case of two users. In the case of more than two users, we outline an iterative algorithm that is of the same complexity order as [4] but conceptually simpler.

\section{Problem Formulation}

The linear downlink problem is illustrated in Fig. 1 and can be formulated as follows: The source variables $X_{i}, i=$ $1, \ldots, K$, are to be conveyed to the corresponding $i$ th receiver, where $K$ is the number of users. The encoder multiplies each source variable with a beamforming vector, $\boldsymbol{u}_{i} \in \mathbb{C}^{N_{T}}$, and a power scaling variable, $\sqrt{P_{i}}$, and transmits the sum $\boldsymbol{\alpha}(\boldsymbol{X})=\sum_{i=1}^{K} \sqrt{P_{i}} \boldsymbol{u}_{i} X_{i}$. The source variables, $X_{i}$, are i.i.d. circularly-symmetric complex Gaussian random variables with unit variance and zero mean, that is, $X_{i} \sim \mathcal{C N}(0,1)$. The beamforming vectors are of unit norm and the power scaling variables fulfill the relation $\sum P_{i} \leq P$, where $P$ is the total average power that can be used by the base station. The conjugated channel to user $i$ is described by the vector $\boldsymbol{h}_{i} \in \mathbb{C}^{N_{T}}$ such that the received signal can be written as

$$
Y_{i}=\sum_{j=1}^{K} \sqrt{P_{j}} h_{i}^{H} \boldsymbol{u}_{j} X_{j}+N_{i}
$$

where $N_{i} \sim \mathcal{C N}(0,1)$ is additive white Gaussian noise (AWGN). The signal-to-interference-and-noise ratio (SINR) can now be expressed as $\operatorname{SINR}_{i}=\frac{P_{i} \boldsymbol{h}_{i}^{H} \boldsymbol{u}_{i} \boldsymbol{u}_{i}^{H} \boldsymbol{h}_{i}}{\sum_{j \neq i} P_{j} \boldsymbol{h}_{i}^{H} \boldsymbol{u}_{j} \boldsymbol{u}_{j}^{H} \boldsymbol{h}_{i}+1}$ and the MSE of the linear minimum MSE (MMSE) estimator $\hat{X}_{i}=E\left[X_{i} \mid Y_{i}\right]$, expressed in terms of the SINR [5], becomes

$$
\begin{aligned}
\mathrm{MSE}_{i} & =E\left[\left|X_{i}-\hat{X}_{i}\right|^{2}\right]=\frac{E\left[\left|X_{i}\right|^{2}\right]}{1+\mathrm{SINR}_{i}} \\
& =1-\frac{P_{i} \boldsymbol{h}_{i}^{H} \boldsymbol{u}_{i} \boldsymbol{u}_{i}^{H} \boldsymbol{h}_{i}}{\sum_{j=1}^{K} P_{j} \boldsymbol{h}_{i}^{H} \boldsymbol{u}_{j} \boldsymbol{u}_{j}^{H} \boldsymbol{h}_{i}+1} .
\end{aligned}
$$




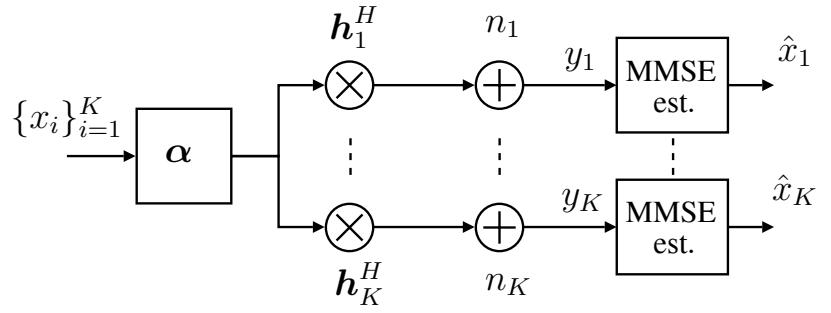

Fig. 1. Downlink transmission. The source $x_{i}$ is transmitted to user $i$, where the vector $\boldsymbol{h}_{i}$ models the channel from the base station to the user. The transmission is disturbed by the additive noise $n_{i}$.

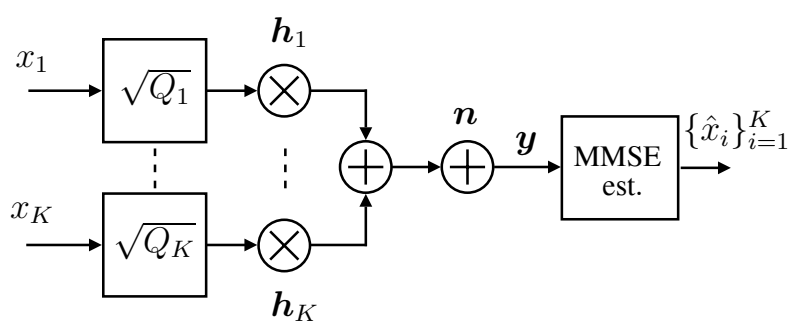

Fig. 2. Dual uplink problem. The source variable $x_{i}$ is transmitted from user $i$ over a MAC, where the vector $\boldsymbol{h}_{i}$ models the channel from the user to the base station. The transmission is disturbed by the additive noise $\boldsymbol{n}$.

We would like to find the jointly optimal beamforming vectors $\left\{\boldsymbol{u}_{i}\right\}$ and power allocation variables $\left\{P_{i}\right\}$ such that the following sum-MSE is minimized

$$
\mathrm{MSE}=\sum_{i=1}^{K} \mathrm{MSE}_{i}
$$

This is a very hard problem due to the fact that even though the power allocation is fixed, the optimal beamforming vector for user $i$ depends on the beamforming vectors to all other users in a complicated manner, as seen from (2) and (3).

\section{DUAL UPLINK FORMULATION}

In [2], it was shown that the problem of SINR balancing can be solved by first solving a dual uplink problem. In the uplink formulation, each optimal receive beamforming vector is independent of the other beamforming vectors and can therefore easily be found for a given power allocation. Due to the close relationship between SINR and MSE, the duality naturally extends to MSE minimization [3].

The dual uplink problem can be formulated as follows: Each user has a source variable $X_{i} \sim \mathcal{C N}(0,1), i=1, \ldots, K$, that is to be conveyed to the base station. At the user node, the source variable is multiplied by a power scaling variable, $\sqrt{Q}_{i}$, and transmitted to the base station over a multiple-access channel (MAC) as seen in Fig. 2. In this formulation, the entries in the channel vector $\boldsymbol{h}_{i} \in \mathbb{C}^{N_{T}}$ model the paths from the $i$ th user to each of the $N_{T}$ antennas at the base station. As before, the transmission is disturbed by an AWGN term $\boldsymbol{N} \sim \mathcal{C} \mathcal{N}(0, \boldsymbol{I})$. The received signal at the base station can be expressed as

$$
\boldsymbol{Y}=\sum_{i=1}^{K} \sqrt{Q_{i}} \boldsymbol{h}_{i} X_{i}+\boldsymbol{N}
$$

where the power scaling variables should fulfill the constraint $\sum Q_{i} \leq P$. Each transmitted source variable is now estimated by using the linear beamforming vectors $\boldsymbol{u}_{i} \in \mathbb{C}^{N_{T}},\left\|\boldsymbol{u}_{i}\right\|=1$, and a scalar scaling $\gamma_{i}$ such that $\hat{X}_{i}=E\left[X_{i} \mid \boldsymbol{Y}\right]=\gamma_{i} \boldsymbol{u}_{i}^{H} \boldsymbol{Y}$. Thus, the SINR of the $i$ th source variable is

$$
\operatorname{SINR}_{i}=\frac{Q_{i} \boldsymbol{u}_{i}^{H} \boldsymbol{h}_{i} \boldsymbol{h}_{i}^{H} \boldsymbol{u}_{i}}{\boldsymbol{u}_{i}^{H}\left(\sum_{j \neq i} Q_{j} \boldsymbol{h}_{j} \boldsymbol{h}_{j}^{H}+\boldsymbol{I}\right) \boldsymbol{u}_{i}}
$$

and the MSE can be obtained in a similar manner as in (2).

Assuming that an arbitrary power allocation $Q=$ $\left(Q_{1}, \ldots, Q_{K}\right)$ (i.e., not necessarily fulfilling the power constraint) is given, the uplink formulation makes it possible to minimize the sum-MSE by individually maximizing each user's SINR. This is done by either of the following two equivalent choices of beamforming vectors [6, Ch. 3]

$$
\begin{aligned}
\boldsymbol{u}_{i}^{\mathrm{opt}}(\boldsymbol{Q}) & =\frac{\left(\sum_{j \neq i} Q_{j} \boldsymbol{h}_{j} \boldsymbol{h}_{j}^{H}+\boldsymbol{I}\right)^{-1} \boldsymbol{h}_{i}}{\left\|\left(\sum_{j \neq i} Q_{j} \boldsymbol{h}_{j} \boldsymbol{h}_{j}^{H}+\boldsymbol{I}\right)^{-1} \boldsymbol{h}_{i}\right\|} \\
& =\frac{\left(\sum_{j=1}^{K} Q_{j} \boldsymbol{h}_{j} \boldsymbol{h}_{j}^{H}+\boldsymbol{I}\right)^{-1} \boldsymbol{h}_{i}}{\left\|\left(\sum_{j=1}^{K} Q_{j} \boldsymbol{h}_{j} \boldsymbol{h}_{j}^{H}+\boldsymbol{I}\right)^{-1} \boldsymbol{h}_{i}\right\|} .
\end{aligned}
$$

By inserting the optimal beamforming vector from (6) into (5) and using the relation between SINR and MSE, we obtain

$$
\operatorname{MSE}_{i}^{\mathrm{opt}}(\boldsymbol{Q})=\frac{1}{1+Q_{i} \boldsymbol{h}_{i}^{H}\left(\sum_{j \neq i} Q_{j} \boldsymbol{h}_{j} \boldsymbol{h}_{j}^{H}+\boldsymbol{I}\right)^{-1} \boldsymbol{h}_{i}} .
$$

\section{A. Properties of Sum-MSE Minimization}

The duality relation is such that an MSE point which is achievable in the uplink can also be achieved in the downlink by using the same beamforming vectors [3]. The uplink/downlink power allocations are in general not equal. Yet, in the special case of sum-MSE minimization, it turns out that also the optimal power allocations are equal, that is,

$$
P_{i}^{\mathrm{opt}}=Q_{i}^{\mathrm{opt}} \quad i=1, \ldots, K .
$$

This relation stems from the fact that the optimal solution is characterized by the relation

$$
\boldsymbol{h}_{i}^{H} \boldsymbol{u}_{j}^{\mathrm{opt}}\left(\boldsymbol{Q}^{\mathrm{opt}}\right)=\left(\boldsymbol{h}_{j}^{H} \boldsymbol{u}_{i}^{\mathrm{opt}}\left(\boldsymbol{Q}^{\mathrm{opt}}\right)\right)^{*} \quad \forall i, j .
$$

A proof of (9) and (10) was recently presented in [7], where the Karush-Kuhn-Tucker conditions are used to prove the relations. Although (9) and (10) involves $Q^{\mathrm{opt}}$, it is not explicit how to find the actual value of $Q^{\text {opt }}$ without the use of numerical methods. The operational meaning of (10) is that the uplink problem not only is dual to the downlink problem, but at the optimal solutions, the problems are identical.

\section{TWO-USER CLOSED-FORM SOLUTION}

We will now use (9) and (10) to derive a closed-form expression for the optimal power allocation in the two-user case. The solution is divided into four cases depending on whether the channels have the same norm and if they are parallel. 
Proposition 1. Without loss of generality, let $\left\|\boldsymbol{h}_{1}\right\| \geq\left\|\boldsymbol{h}_{2}\right\|>$ 0. The optimal uplink/downlink power allocation in the twouser sum-MSE case is given by

$$
P_{1}^{\text {opt }}= \begin{cases}\min \left(\tilde{P}_{1}, P\right) & \text { if } a_{2} \neq 0 \text { and } a_{3} \neq 0, \\ P & \text { if } a_{2} \neq 0 \text { and } a_{3}=0, \\ P / 2 & \text { if } a_{2}=0 \text { and } a_{3} \neq 0, \\ {[0, P]} & \text { if } a_{2}=0 \text { and } a_{3}=0,\end{cases}
$$

where

$$
\begin{aligned}
& a_{1}=2+P\left\|\boldsymbol{h}_{2}\right\|^{2}, \quad a_{2}=\left\|\boldsymbol{h}_{1}\right\|^{2}-\left\|\boldsymbol{h}_{2}\right\|^{2} \geq 0, \\
& a_{3}=\left\|\boldsymbol{h}_{1}\right\|^{2}\left\|\boldsymbol{h}_{2}\right\|^{2}-\left|\boldsymbol{h}_{1}^{H} \boldsymbol{h}_{2}\right|^{2} \geq 0, \\
& \tilde{P}_{1}=\frac{-a_{1}+\sqrt{a_{1}^{2}+P a_{1} a_{2}+a_{2}^{2} / a_{3}}}{a_{2}} .
\end{aligned}
$$

Proof: Starting with the inner product in (10), we have

$$
\begin{aligned}
& \boldsymbol{h}_{2}^{H} \boldsymbol{u}_{1}^{\mathrm{opt}}\left(\boldsymbol{P}^{\mathrm{opt}}\right) \stackrel{(a)}{=} \frac{\boldsymbol{h}_{2}^{H}\left(P_{2}^{\mathrm{opt}} \boldsymbol{h}_{2} \boldsymbol{h}_{2}^{H}+\boldsymbol{I}\right)^{-1} \boldsymbol{h}_{1}}{\left\|\left(P_{2}^{\mathrm{opt}} \boldsymbol{h}_{2} \boldsymbol{h}_{2}^{H}+\boldsymbol{I}\right)^{-1} \boldsymbol{h}_{1}\right\|} \\
& \stackrel{(b)}{=} \frac{\boldsymbol{h}_{2}^{H}\left(\boldsymbol{I}-\frac{P_{2}^{\mathrm{opt}} \boldsymbol{h}_{2} \boldsymbol{h}_{1}^{H}}{1+P_{2}^{\mathrm{opt}} \boldsymbol{h}_{2}^{H} \boldsymbol{h}_{2}}\right) \boldsymbol{h}_{1}}{\left\|\left(\boldsymbol{I}-\frac{P_{2}^{\mathrm{opt}} \boldsymbol{h}_{2} \boldsymbol{h}_{2}^{H}}{1+P_{2}^{\mathrm{opt}} \boldsymbol{h}_{2}^{H} \boldsymbol{h}_{2}}\right) \boldsymbol{h}_{1}\right\|} \\
& \stackrel{(c)}{=} \frac{\boldsymbol{h}_{2}^{H}\left(\left(1+P_{2}^{\mathrm{opt}} \boldsymbol{h}_{2}^{H} \boldsymbol{h}_{2}\right) \boldsymbol{I}-P_{2}^{\mathrm{opt}} \boldsymbol{h}_{2} \boldsymbol{h}_{2}^{H}\right) \boldsymbol{h}_{1}}{\left\|\left(\left(1+P_{2}^{\mathrm{opt}} \boldsymbol{h}_{2}^{H} \boldsymbol{h}_{2}\right) \boldsymbol{I}-P_{2}^{\mathrm{opt}} \boldsymbol{h}_{2} \boldsymbol{h}_{2}^{H}\right) \boldsymbol{h}_{1}\right\|} \\
& \stackrel{(d)}{=} \frac{\boldsymbol{h}_{2}^{H} \boldsymbol{h}_{1}}{\left\|\left(\left(1+P_{2}^{\mathrm{opt}} \boldsymbol{h}_{2}^{H} \boldsymbol{h}_{2}\right) \boldsymbol{I}-P_{2}^{\mathrm{opt}} \boldsymbol{h}_{2} \boldsymbol{h}_{2}^{H}\right) \boldsymbol{h}_{1}\right\|} \\
& \stackrel{(e)}{=} \frac{\left(\boldsymbol{h}_{1}^{H} \boldsymbol{h}_{2}\right)^{*}}{\left\|\left(\left(1+P_{1}^{\mathrm{opt}} \boldsymbol{h}_{1}^{H} \boldsymbol{h}_{1}\right) \boldsymbol{I}-P_{1}^{\mathrm{opt}} \boldsymbol{h}_{1} \boldsymbol{h}_{1}^{H}\right) \boldsymbol{h}_{2}\right\|},
\end{aligned}
$$

where (a) follows from the definition of $\boldsymbol{u}_{1}^{\text {opt }}$ in (6), (b) from using the matrix inversion lemma, (c)-(d) are basic manipulations, and (e) follows from the symmetry in (10). Since the numerators in (13) and (14) are equal we must have that also the denominators are equal. By using this equality, squaring both sides and using the relation $P=P_{1}^{\text {opt }}+P_{2}^{\text {opt }}$, we get a second-order equation, which when solved gives the expression in (12). Since the solution to a second-order equation in general is unbounded, we need $\min \left(\tilde{P}_{1}, P\right)$ to make sure that the power usage does not exceed the power limit. In the final expression in (11), we also have to consider the cases when $a_{2}$ or $a_{3}$ are equal to zero. $a_{3}=0$ corresponds to the channels being parallel, in which case the optimal (linear) strategy is to allocate all power to the user with the strongest channel. $a_{2}=0$ on the other hand corresponds to the case where the two channels have the same norm, in which case the power should be divided equally among the users. $P_{1}^{\text {opt }}$ is continuous in the special cases mentioned above and can also be found by taking the limit of $\tilde{P}_{1}$ as $a_{2}$ or $a_{3}$ approaches zero. If we have $a_{2}=a_{3}=0$, it can be shown by inserting (2) into (3) that the sum-MSE is invariant to the power allocation and only dependent on the sum.

The power allocation for the second user is easily found since $P=P_{1}^{\mathrm{opt}}+P_{2}^{\mathrm{opt}}$. Once the optimal power allocation has been determined, the optimal beamforming vectors can be calculated using (6) or (7). For users that are assigned zero power, the beamforming vectors can be arbitrarily chosen. The relation in (10) is therefore not necessarily fulfilled for these users.

\section{V. $K>2$ USERS}

A generalization to $K>2$ users is not straightforward and finding a closed-form solution for this case is still an open problem. We briefly outline an efficient iterative algorithm that takes advantage of the relation in (10). By inserting (7) into (10) and defining $\boldsymbol{A} \triangleq \sum_{i=1}^{K} P_{i} \boldsymbol{h}_{i} \boldsymbol{h}_{i}^{H}+\boldsymbol{I}$, it can be shown that, for the optimal power allocation, $C_{j}=\left\|\boldsymbol{A}^{-1} \boldsymbol{h}_{j}\right\|=C$ is constant for all $j$. Given an initial power allocation (e.g., uniform), we propose an algorithm that is based on evaluating $C_{j}$ for all users and calculating the arithmetic mean $\bar{C}$ for users with positive power allocation. Next, the power allocation $P_{j}$ is increased proportionally to $C_{j}-\bar{C}$ for all users. By updating the powers iteratively, we have been able to find the optimal power allocation for systems with hundreds of users. In each step, one has to take care so that no user has negative power, in which case the power is set to zero, and that the power constraint is fulfilled. The complexity of the algorithm is of the same order as the iterative algorithm presented in [4], that is, $\mathcal{O}\left(L\left(N_{T}^{3}+K N_{T}^{2}\right)\right)$, where $L$ is the number of iterations. In comparison, algorithms based on convex optimization have a complexity of $\mathcal{O}\left(K^{6.5} N_{T}^{6.5}\right)$ [3].

\section{CONCLUSIONS}

We have considered sum-MSE minimization for the Gaussian MIMO broadcast channel. By using recently discovered properties of this problem, we have derived a closed-form expression for the optimal power allocation in the two-user scenario and proposed a conceptually simple and efficient algorithm that handles an arbitrary number of users. It is not clear how or whether the closed-form solution can be generalized to more than two users. Our hope is that the solution we present can inspire future research on the more general case.

\section{REFERENCES}

[1] H. Weingarten, Y. Steinberg, and S. Shamai, "The capacity region of the Gaussian multiple-input multiple-output broadcast channel," IEEE Trans. Inf. Theory, vol. 52, no. 9, pp. 3936-3964, Sep. 2006.

[2] M. Schubert and H. Boche, "Solution of the multiuser downlink beamforming problem with individual SINR constraints," IEEE Trans. Veh. Technol., vol. 53, no. 1, pp. 18-28, Jan. 2004.

[3] S. Shi, M. Schubert, and H. Boche, "Downlink MMSE transceiver optimization for multiuser MIMO systems: duality and sum-MSE minimization," IEEE Trans. Signal Process., vol. 55, no. 11, pp. 5436-5446, 2007.

[4] A. Mezghani, M. Joham, R. Hunger, and W. Utschick, "Transceiver design for multi-user MIMO systems," in Int. ITG WSA, 2006.

[5] P. Viswanath, V. Anantharam, and D. N. C. Tse, "Optimal sequences, power control, and user capacity of synchronous CDMA systems with linear MMSE multiuser receivers," IEEE Trans. Inf. Theory, vol. 45, no. 6, pp. 1968-1983, 1999.

[6] R. A. Monzingo and T. W. Miller, Introduction to Adaptive Arrays. SciTech Publishing, 2004.

[7] A. J. Tenenbaum and R. S. Adve, "Minimizing sum-MSE implies identical downlink and dual uplink power allocations," IEEE Trans. Commun., vol. 59, no. 3, pp. 686-688, Mar. 2011. 